\title{
Identification of early biomarkers in a rabbit model of primary Candida pneumonia
}

\author{
Gang $\mathrm{Lu}^{1 \dagger}$, Chen Wang ${ }^{2 \dagger}$, Chunrong Wu', Lei Yan ${ }^{1}$ and Jianguo Tang ${ }^{1 *}$ (D)
}

\begin{abstract}
Background: Candida albicans is an opportunistic pathogen, but since it also belongs to the normal fungal flora, positive sputum culture as the solely basis for the diagnosis of invasive Candida albicans pneumonia can easily lead to excessive antifungal therapy. Therefore, identification of a pneumonia biomarker might improve diagnostic accuracy.

Methods: A rabbit model was established by inoculating $5 \times 10^{7} \mathrm{cfu} / \mathrm{mL}$ C. albicans into the trachea of 20 rabbits with 20 rabbits as control group. Infection was monitored by chest thin-layer computed tomography (CT). $2 \mathrm{~mL}$ blood samples were collected daily during each infection and serum levels of potential biomarkers were measured by enzyme-linked immunosorbent assay (ELISA). Seven-day post-inoculation the rabbits were sacrificed by $\mathrm{CO}_{2}$ asphyxiation and lung tissue was histopathologically examined and blood was brought to culture. Data were statistically analyzed.

Results: Infection became evident as early as day 3 post-inoculation. The levels of soluble triggering receptor expressed on myeloid cells-1 (sTREM-1), soluble hemoglobin-haptoglobin scavenger receptor (sCD163), procalcitonin (PCT) and tumor necrosis factor-a (TNF-a) were elevated in the experimental group compared to the control $(P<0.01)$, whereas the levels of C-reactive protein (CRP), interleukin-6 (IL-6), IL-8 and IL-10 showed no significant differences $(P>0.05)$. The dynamic curves of the levels of CRP, IL-6, IL-8, IL-10, SCD163 and TNF- $a$ in both groups demonstrated a similar trend during infection but differences between the groups was observed only in the STREM-1 levels. Receiver-operating characteristics (ROC) curve analysis showed that the sensitivity and specificity were 85 and $80 \%$ for sTREM-1 (cut-off value: $45.88 \mathrm{pg} / \mathrm{mL}$ ) and 80 and $75 \%$ for SCD163 (cut-off value: $16.44 \mathrm{U} / \mathrm{mL}$ ), respectively. The values of the area under the ROC curve (AUC ROC) of STREM-1 and SCD163 were 0.882 (95\% Cl: 0.922-0.976) and 0.814 (95\% Cl: 0.678-0.950), respectively. Other markers did not exhibit significant differences.
\end{abstract}

Conclusion: STREM-1 and SCD163 might be suitable biomarkers for pneumonia.

Keywords: sTREM-1, Candida albicans, Lung infection, Biomarkers, sTREM-1, SCD163

\section{Background}

A serious risk factor for patients in hospital intensive care units (ICUs) is the potential of contracting opportunistic infections from a variety of pathogens. The EPIC II study, which investigated the prevalence of infections in 1265 ICUs across 75 countries, showed that $51 \%$ of

\footnotetext{
* Correspondence: tangjianguo@5thhospital.com

${ }^{\dagger}$ Gang Lu and Chen Wang contributed equally to this work.

'Department of Trauma-Emergency \& Critical Care Medicine, Shanghai Fifth People's Hospital, Fudan University, No. 128 Ruili Road, Shanghai 200240, China

Full list of author information is available at the end of the article
}

13,796 cases contracted infections, of which $64 \%$ involved the respiratory system and 19\% of them were caused by fungi [1].

Nevertheless, diagnosis of Candida pneumonia is a challenge, since Candida spp. are part of the normal flora and asymptomatic colonizers of various mucosal surfaces including the upper respiratory tract. There is a need to differentiate between primary isolated Candida lung invasion causing pneumonia and secondary hematogenously disseminated Candida reaching the lungs from elsewhere. Pneumonia caused by fungi is provisionally diagnosed by

(C) The Author(s). 2019 Open Access This article is distributed under the terms of the Creative Commons Attribution 4.0 International License (http://creativecommons.org/licenses/by/4.0/), which permits unrestricted use, distribution, and 
a combination of clinical and radiological findings, together with cultures and serology [2].

To detect the presence of infection and inflammation in patients, a wide variety of potential serum markers were investigated including factors involved in infection and inflammatory responses i.e., soluble triggering receptor expressed on myeloid cells (sTREM-1, a member of the immunoglobulin superfamily) [3-7], soluble hemoglobin-haptoglobin scavenger receptor (sCD163) [8-10], procalcitonin (PCT) [11], C-reactive protein (CRP) [12] as well as interleukin (IL)-6, IL-8, IL-10 and tumor necrosis factor alpha (TNF- $\alpha$ ) [13-16]. However, the majority of studies that investigated biomarkers for infectious diseases focused on a relatively heavy load of septic infection and did not differentiate fungal from bacterial infections and to date only a few studies have focused on C. albicans infection [17]. Therefore, identification of marker for Candida-induced pneumonia (CP) would be highly desirable to facilitate the early diagnosis and effective treatment of patients. In the present study, we established a rabbit CP model and evaluated potential biomarkers (vide supra) using biochemical and statistical analyses for them to be useful for the early diagnosis and prognosis of $\mathrm{CP}$.

\section{Methods}

\section{Animals and microorganisms}

All procedures involving animals were performed in accordance with the ethical standards of the participating institution and the Guidelines for the Humane Treatment of Laboratory Animals (Ministry of Science and Technology of the People's Republic of China, Policy No. 2006 398), and were approved by the Institutional
Animal Care and Use Committee of Shanghai Fifth People's Hospital.

A total of 40, pathogen free, New Zealand white rabbits (males, 2 months old, $2.0-2.5 \mathrm{~kg}$ ) were purchased from the Animal Center of the Shanghai Jiaotong University. The animals were reared in well ventilated, stainless steel, $60 \times 80 \mathrm{~cm}$ rabbit cages ( 2 rabbits per cage) placed in a temperature-controlled room $\left(20 \mathrm{~m}^{2}\right)$ at 20 $30{ }^{\circ} \mathrm{C}$ and $40-70 \%$ humidity. Lighting included a combination of natural light and fluorescence. Tap water and mixed pellet feed were provided daily. Rabbit cages and drinking bottles were sterilized using $0.1 \%$ benzalkonium bromide and bedding was changed every 2 days. The animals were observed for 5 days and used for experimentation in the absence of anomalous behavior.

All animals received $100 \mathrm{mg} / \mathrm{kg}$ cyclophosphamide via an ear vein injection daily from day 1 to 6 preinoculation to maintain a low immune status [18]. Bacterial infection was prevented with $400 \mathrm{mg} / \mathrm{kg}$ cefuroxime from day 4 onwards (Fig. 1). A standard strain of C. albicans (ATCC10235) was used for infection. Figure 1 describes the establishment procedure of the rabbit model.

\section{Establishment of a primary rabbit $\mathrm{CP}$ model}

On day 6 pre-inoculation, rabbits were randomly divided into 2 groups of 20 . The experimental group received a $1 \mathrm{~mL}$ injection of $5 \times 10^{7} \mathrm{cfu} / \mathrm{mL}$ C. albicans solution via percutaneous tracheal puncture, while the control group received normal saline via the same route. The rabbits were anesthetized with $2 \mathrm{~mL} / \mathrm{kg}$ intravenous injection of $3 \%$ sodium pentobarbital and carefully positioned on the surgical operating table. After trimming neck hair and

40 rabbits received $100 \mathrm{mg} / \mathrm{kg}$ cyclophosphamide from pre-inoculation day 1 to 5 and an antibiotic agent ( $400 \mathrm{mg} / \mathrm{kg}$ cefuroxime) from pre-inoculation day 4 . On pre-inoculation day 5 a chest CT was performed.

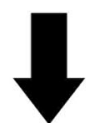

On day 6 rabbits in the experimental group $(n=20)$ received a $1 \mathrm{ml}$ injection with $5 \times 10^{7} \mathrm{cfu} / \mathrm{mL} \mathrm{C}$. albicans solution and rabbits of the control group $(\mathrm{n}=20)$ received $1 \mathrm{~mL}$ saline via percutaneous tracheal punctures.

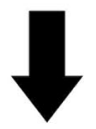

From day 1 to 7 post-inoculation, $2 \mathrm{~mL}$ blood was collected daily via ear vein puncture from rabbits in the experimental and the control groups. Blood was analyzed for the levels of potential biomarkers as well as WBC count and paO2 values. Lung CTs were performed on day 3 and day 7 post-inoculation while blood cultures were prepared on day 7 post-inoculation.

Fig. 1 Establishment procedure of the C. albicans pneumonia rabbit model 
disinfection with iodine, a towel with a hole was placed over the rabbit neck. Using sterile gloves, the position of the trachea was determined, and the trachea was fixed and punctured at a $45^{\circ}$ oblique angle. After inoculation with $1.0 \mathrm{~mL}$ C. albicans solution, the rabbits were immediately moved to a dorsal elevated position and gently shaken for $5 \mathrm{~min}$ to allow the inoculum to enter the lower respiratory tract. Finally, the animals were returned to their respective cages to wake up naturally.

\section{Chest computed tomography imaging}

Rabbits in the experimental and control groups were placed in the prone position after intravenous injection of $3 \%$ pentobarbital sodium $(2 \mathrm{~mL} / \mathrm{kg})$ for chest thinlayer CT on day 1 pre-inoculation and days 3 and 7 post-inoculation (GE light speed 64VCT, USA). Two independent experts evaluated the CT images.

\section{Sample collection and measurements}

From day 1 to 7 post-inoculation, $2 \mathrm{~mL}$ blood samples were collected daily from ear vein punctures of rabbits in the experimental and control groups. The blood gas parameter $\left(\mathrm{PaO}_{2}\right)$ was measured with a gas analysis instrument (GEM Premier 3000, USA), and also white blood cell (WBC) counts in serum. The protein levels of TNF- $\alpha$, sCD163, sTREM-1, PCT, CRP, IL-6, IL-8 and IL10 in serum were measured using ELISA (Guangzhou Jianlun Biological Technology Co., Ltd.). The ELISA kits used were sTREM-1 (96 T kit, JL003211), PCT (JL002421), IL-6 (JL002052), IL-8 (JL001248), IL-10 (JL001132) and TNF- $\alpha$ (JL004161). ELSA analysis was performed according to the manufacturer instructions.

\section{Pathological analysis of rabbit lung tissue}

On day 7 post-inoculation, the rabbits were euthanized with carbon dioxide $\left(\mathrm{CO}_{2}\right)$ in a $\mathrm{CO}_{2}$ chamber and lung tissue biopsy was performed while blood samples were cultured to detect whether C. albicans was present. The neck muscles were blunt dissected and the trachea dissociated to separate lung from thorax on a sterile console. Lung tissue was visually observed for pathological changes, followed by collection of pathological specimens of lung tissue with the dimension $2.0 \times 2.0 \times 0.2$ to $0.3 \mathrm{~cm}$ from each pulmonary lobe near the airways and specimens from the surrounding lung tissue area with visually obvious lesions or palpable nodules. Tissue samples were fixed by immersion in a 4 -fold volume of $10 \%$ formalin and processed for periodic acid Schiff (PAS) and hematoxylin and eosin (HE) staining.

\section{Detection of pulmonary infection and CP diagnosis with thin layer CT scans}

Rabbit lungs were examined for $C$. albicans infection using prone position thin $\mathrm{CT}$ scans after 3 and 7 days of inoculation. CP diagnostic imaging was graded as mild, moderate or severe based on comparing the lung CT images before inoculation and 7 days later. The actual lesion area covering the entire lobe or integration of multiple nodules close to or $>1$ lobe was defined as severe; actual lesion area or the area after integration of multiple nodules $<1$; more than $1 / 2$ of the lung lobe involved was defined as moderate; nodules or actual lesion area $<1 / 2$ of the lung lobe, or small patches shadow, circumscribed bronchopneumonia shadow was defined as mild. Finally, 7 rabbits were evaluated as mild CP, 6 rabbits were in the moderate $\mathrm{CP}$ group and 7 rabbits were in the severe group. Finally $\mathrm{CP}$ was also diagnosed based on the presence of C. albicans forms and pseudohyphae/ hyphae in lung tissue visualized by PAS staining.

\section{Statistical analysis}

SPSS ver. 16.0 software (SPSS, Chicago, Illinois, USA) was used to perform all statistical analyses. The WBC count and oxygenation index with normal distributions are reported as the mean $\pm \mathrm{SD}$. A Mann-Whitney U test was used to compare non-normally distributed data such as PCT, SCD163, sTREM-1, CRP, IL-6, IL-8, IL10 , and TNF- $\alpha$ levels and the results are expressed as median values (interquartile range). Normally distributed data from appropriate groups were compared using a $t$-test. Qualitative data are presented as proportions. Comparisons between the 2 groups were made using a chi-squared test. The Spearman correlation coefficient was used to determine the coefficient for each biomarker investigated. The values of the area under the ROC (receiver-operating characteristics) curves $\left(A U C_{R O C}\right)$ were used to establish the specificity and sensitivity of each biomarker. All $P$-values were 2 -sided and $P$-values $<0.05$ were considered to be statistically significant.

\section{Results \\ Establishment of a rabbit primary CP model}

To establish a rabbit primary CP model, animals were immunosuppressed with cyclophosphamide and bacterial infection was prevented by the administration of cefuroxime. C. albicans was inoculated into rabbits in the experimental group and the resulting infection was monitored by blood culture, lung biopsy and chest CT. Blood cultures from rabbits in the experimental $(n=20)$ and the control $(\mathrm{n}=20)$ groups were both negative for C. albicans. However, C. albicans was detected on histopathology and cultures from lungs of rabbits in the experimental but not in the control group. No pathogenic bacteria were detected in all cultures.

On day 7 after C. albicans infection, lung histopathologic analysis was performed. In lungs isolated from the infected group, white diffuse nodular lesions were visually 
observed (Fig. 2a, b and c), while lungs from the control group showed no sign of infection (Fig. 2Aa). PAS staining of the infected lung tissue specimens showed pseudohyphae/hyphae appearing as thin and straight structures (white arrows) and round and oval yeast forms dyed purple and brown (black arrows) (Fig. 2Bb). H\&E staining showed infiltrating lymphocytes (blue arrows) (Fig. 2Ca) and infiltration of eosinophilic granulocytes (yellow arrows) (Fig. 2Cb).

On days 3 and 7 post-inoculation, chest thin-layer CT demonstrated progressive or stable lung lesions in all animals in the experimental group (Fig. $3 \mathrm{c}$ and d), while infection was absence in the control group (Fig. 3a and b). These observations showed that the rabbit $\mathrm{CP}$ model had been successfully established.

\section{Correlation analysis between biomarkers and the severity} levels of $\mathrm{CP}$

During the first week after inoculation with C. albicans, blood samples were collected from rabbits in the experimental and control groups. Since lung CT scans showed signs of pulmonary infection as early as day 3 post-inoculation, we analyzed blood samples collected on day 3 to evaluate the levels of several potential biomarkers, as well as WBC counts. To assess respiratory function, $\mathrm{PaO}_{2}$ levels were measured. The results showed no significant difference in CRP levels between the control and mild experimental groups. However, as the disease worsened, the CRP level in the experimental group increased significantly and was significantly higher in the severe group than

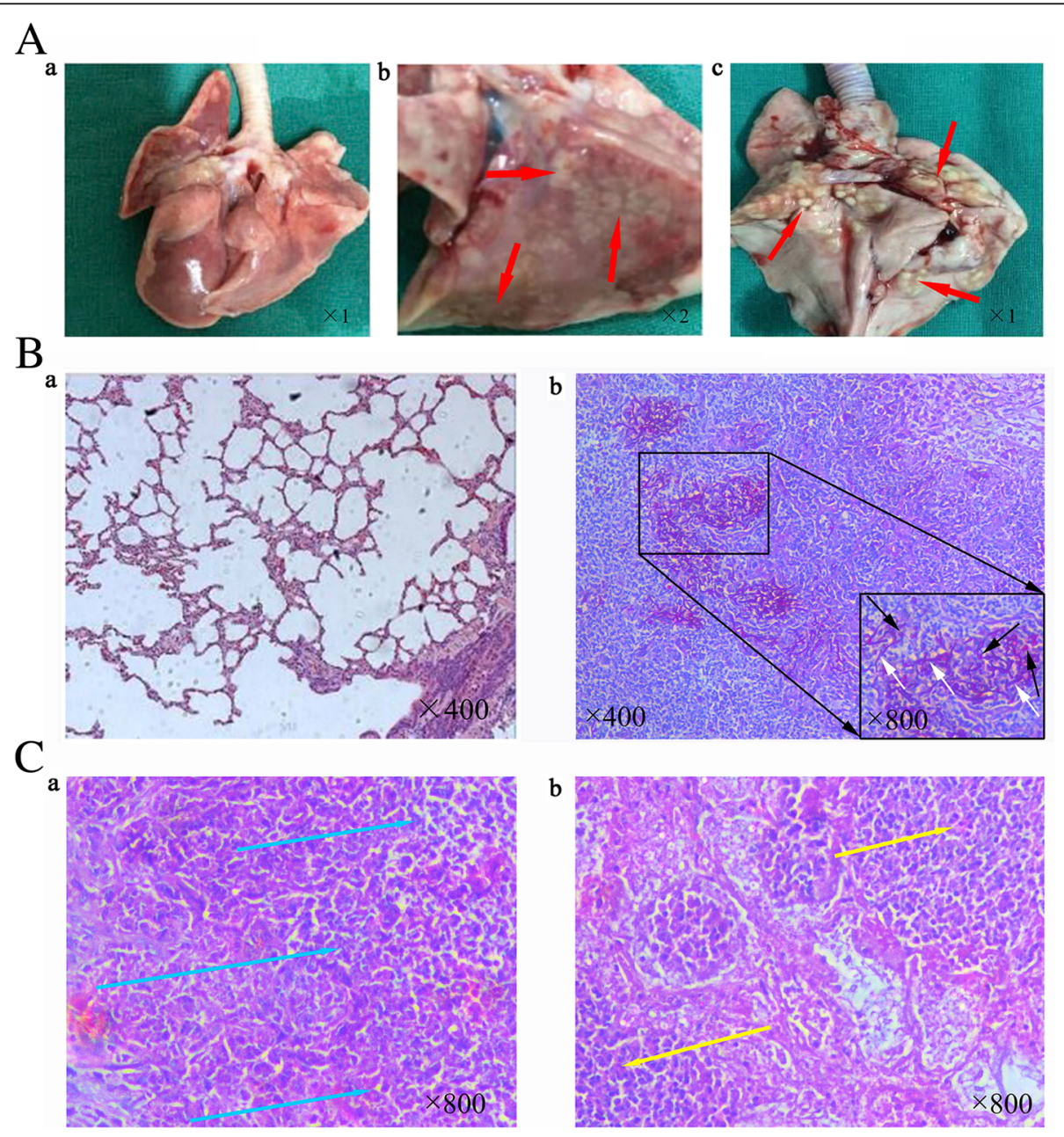

Fig. 2 Macroscopic and histopathology of rabbit lung specimens. On the 7th day after C. albicans infection, lung specimens were visually examined. a) Histopathological images of (a) a normal healthy lung specimen from the control group (magnification $\times 1$ ), (b) a lung from the $C$. albicans infection model group with nodular lesions (red arrows) (magnification $\times 2$ ), (c) a lung from the rabbit C. albicans infection model group with diffuse nodular lesions (red arrows) (magnification $\times 1$ ). B) PAS staining of (a) a normal healthy lung tissue and (b) upper panel, a lung tissue of a rabbit infected with C. albicans (magnification $\times 400$ ), (b) lower panel, white arrows indicate pseudohyphae/hyphae, black arrows indicate yeast forms (magnification $\times 800$ ). c) HE staining of $(\mathbf{a})$ infiltration of surrounding lymphocytes (blue arrows) and (b) infiltration of surrounding eosinophilic granulocyte (yellow arrows) (magnification $\times 800$ ) 

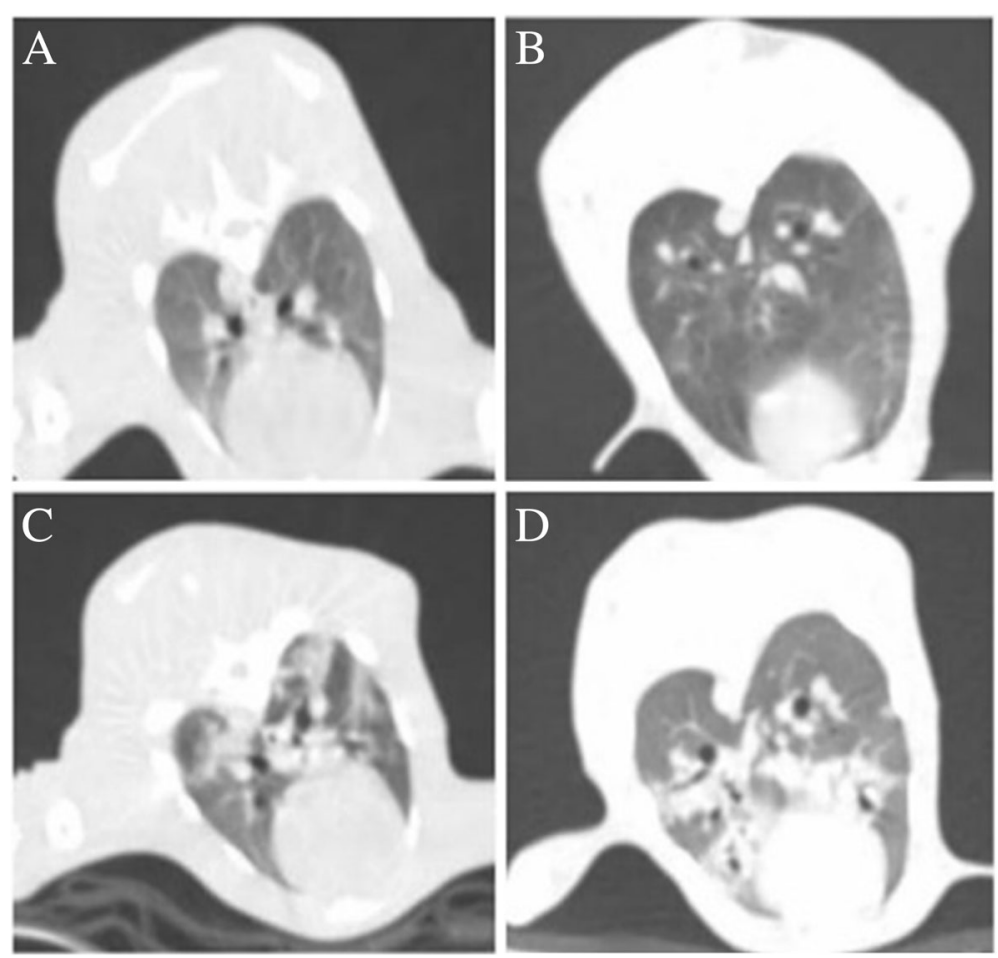

Fig. 3 Chest CT images of a normal rabbit and a rabbit with severe white Candida pneumonia. (a and $\mathbf{b})$ normal rabbit, (c) Day 3 postinoculation demonstrating patchy shadows, substantial lesions and nodules, (d) Day 7 post-inoculation demonstrating larger lesions in the entire lung as compared to day 3 post-inoculation

that in mild group $(P<0.01)$. The difference in IL-6 values between the control and experimental groups was significant, but this difference did not reflect the severity of pneumonia. There was no significant difference in IL-8 levels between the control and experimental groups. The IL-8 level did not increase with the severity of pneumonia. There was a significant difference in IL-10 levels between the control and experimental groups, but these did not reflect the severity of pneumonia. There was a significant difference in PCT levels between the control and experimental groups, when the PCT level increased with increasing severity of pneumonia, with a significantly higher level in the severe group compared with the mild group $(P<0.01)$. SCD163 levels were significantly higher in the experimental group than in control $(P<0.01)$. The values of sTREM1 and TNF- $\alpha$ were higher in the experimental group than in the control group, but they did not increase with the severity of pneumonia. The WBC count was higher in the mild group than in control, but lower in the severe group compared with the control group. Thus, the WBC count did not reflect the infection and its severity. The $\mathrm{PaO}_{2}$ level was significantly lower in the experimental group than in the control group, which gradually decreased along with aggravation of pneumonia (Fig. 4).
Dynamic curves of the serum levels of individual markers during infection

To investigate further these markers, their serum levels were plotted against the pre- and post-inoculation times (days 2, 3, 4, 5, 6 and 7) to create dynamic curves. The curves of the levels of TNF- $\alpha$, CRP, IL- 6 , IL- 8 and IL-10, PCT, SCD163, as well as WBC counts, showed a similar trend in the experimental and control groups (Fig. 5). However, despite a similarity of the trend of the curve, significant differences between the experimental and the control groups in the levels of sCD163 $(P=0.002)$, TNF$\alpha(P=0.017)$, and sTREM-1 $(P=0.001)$, in addition to PCT $(P=0.037)$ were measured as early as day 3 post-inoculation. However, the interquartile ranges were very large for PCT, TNF- $\alpha$ and sCD163.

A significantly different trend was found in the sTREM-1 levels, with the levels in both groups gradually increasing immediately after inoculation and then declining until day 4. However, the levels in the experimental group started to increase thereafter, while the levels in the control group remained constant (Fig. 5), indicating a high potential of sTREM-1 as a biomarker.

\section{ROC curve analyses of biomarkers}

To determine the specificity and sensitivity of biomarkers, ROC curve analyses were carried out (Table 1 

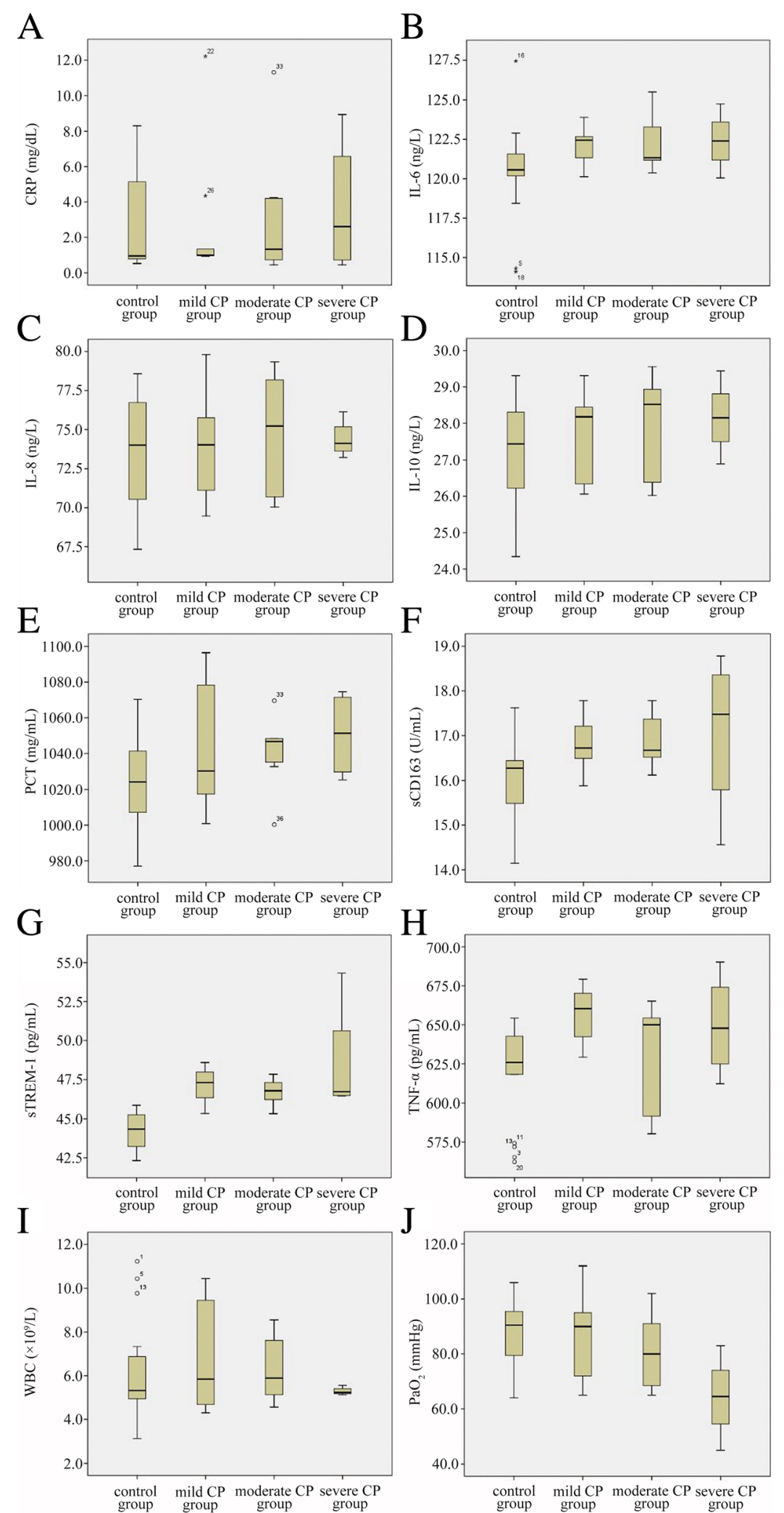

Fig. 4 Correlation analysis between biomarkers and severity levels of CP (a) CRP; (b) IL-6; (c) IL-8; (d) IL-10, (e) PCT; (f) SCD163; (g) sTREM-1; (h) TNF-a; (i) WBC; (j) $\mathrm{PaO}_{2}$ 


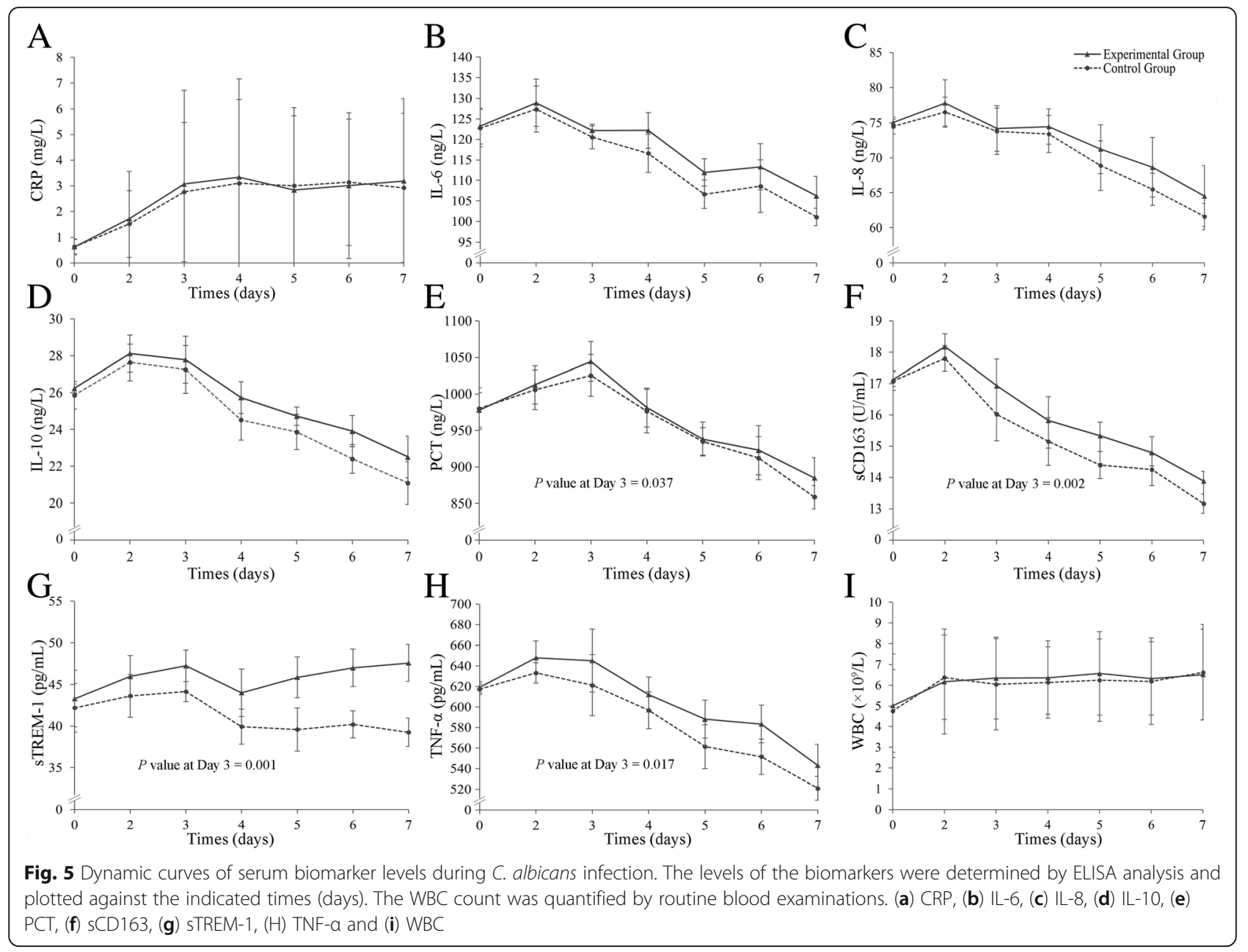

Table 1 ROC curve analysis for potential biomarkers for CP diagnosis

\begin{tabular}{lllll}
\hline & AUC $(95 \% \mathrm{Cl})$ & Cut-off value & Sensitivity & Specificity \\
\hline PCT & $0.685(0.420,0.850)$ & $1027.84 \mathrm{ng} / \mathrm{L}$ & $75 \%$ & $65 \%$ \\
sCD163 & $0.814(0.678,0.950)$ & $16.44 \mathrm{U} / \mathrm{mL}$ & $80 \%$ & $75 \%$ \\
sTREM-1 & $0.882(0.922,0.976)$ & $45.88 \mathrm{pg} / \mathrm{mL}$ & $85 \%$ & $80 \%$ \\
CRP & $0.55(0.363,0.737)$ & $0.944 \mathrm{mg} / \mathrm{L}$ & $70 \%$ & $55 \%$ \\
$\mathrm{IL}-6$ & $0.724(0.563,0.844)$ & $121.26 \mathrm{ng} / \mathrm{L}$ & $70 \%$ & $60 \%$ \\
$\mathrm{IL}-8$ & $0.514(0.333,0.702)$ & $73.63 \mathrm{ng} / \mathrm{L}$ & $60 \%$ & $50 \%$ \\
IL-10 & $0.620(0.444,0.796)$ & $27.50 \mathrm{ng} / \mathrm{L}$ & $60 \%$ & $55 \%$ \\
TNF-a & $0.74(0.583,0.897)$ & $638.89 \mathrm{pg} / \mathrm{mL}$ & $70 \%$ & $60 \%$ \\
WBC & $0.553(0.371,0.734)$ & $5.38 \times 10^{9} / \mathrm{L}$ & $55 \%$ & $55 \%$ \\
$\mathrm{PaO}_{2}$ & $0.333(0.159,0.506)$ & $78 \mathrm{mmHg}$ & $50 \%$ & $25 \%$ \\
\hline
\end{tabular}

PCT: procalcitonin, $\mathrm{sCD} 163$ : soluble hemoglobin-haptoglobin scavenger receptor, sTREM-1: soluble triggering receptor expressed on myeloid cells-1, CRP: C-reactive protein, IL-6: interleukin-6, IL-8: interleukin-8, IL-10: interleukin10, TNF-a: tumor necrosis factor-a, WBC: white blood cell, $\mathrm{PaO}_{2}$ : blood gas parameter, AUC: value of area under the ROC curve and Fig. 6). When the cut-off value was set as $45.88 \mathrm{pg} /$ $\mathrm{mL}$, the $\mathrm{I}^{2}$ for the sensitivity and specificity of sTREM-1 for $\mathrm{CP}$ diagnosis was 85 and $80 \%$, respectively, and the value of the $\mathrm{AUC}_{\mathrm{ROC}}$ was 0.882 (95\% CI: 0.922-0.976). $\mathrm{I}^{2}$ values for the sensitivity and specificity of $\mathrm{sCD} 163$ (the cut-off value $16.44 \mathrm{U} / \mathrm{mL}$ ) were 80 and $75 \%$, respectively, and $\mathrm{AUC}_{\mathrm{ROC}}$ was 0.814 (95\% CI: 0.678 0.950). As shown in Table 1, sTREM-1 and SCD163 demonstrated higher sensitivity and specificity as well as reliability compared to other candidate markers such as PCT, CRP, TNF- $\alpha$ and IL-6, IL-8 and IL-10. Especially, STREM-1 showed the highest specificity and sensitivity as a biomarker for $\mathrm{CP}$ and its $\mathrm{AUC}_{\mathrm{ROC}}$ value was the closest to 1 .

\section{Discussion}

In the present study a potential value of sTREM-1 and sCD163 has been demonstrated in a rabbit model of Candida pneumonia. It has been reported that STREM-1 levels in the bronchoalveolar lavage fluid of patients with bacterial or fungal pneumonia is significantly higher compared to patients with viral or 

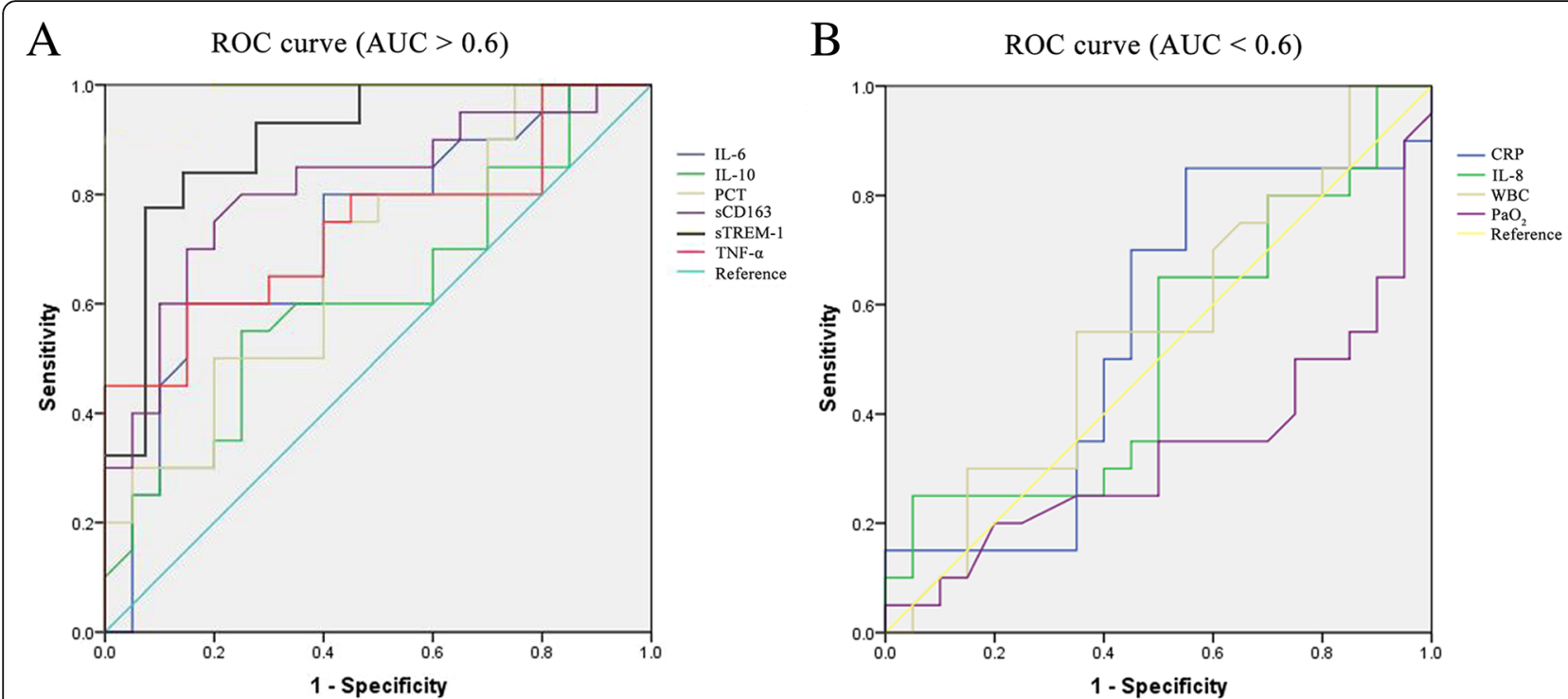

Fig. 6 ROC curves of indicated potential CP biomarkers. (a) Biomarkers with AUC $>0.6$ and (b) biomarkers with AUC $<0.6$

atypical pneumonia, and it has been suggested that sTREM-1 could be used as a potential marker to differentially diagnose pneumonia [19]. In addition, a meta-analysis by Shi et al. suggested that sTREM-1 is a useful biomarker in ICU patients suffering from bacterial lung infections, but that further studies are required to confirm the ideal cut-off value [20]. In an effort to identify specific and sensitive biomarkers for the early diagnosis and prognosis of $\mathrm{CP}$, we first established a rabbit model for primary CP. Mice are frequently used as animal models to study microbial infection because of their cost, ease of handling, technical feasibility and availability of strains [21, 22]. In the present study, we chose rabbits in order to take advantage of larger animals, which allowed us to sample the blood repeatedly for analysis of serum biomarkers and to visualize anatomical details by $\mathrm{CT}$ during the progression of C. albicans infection [23]. Histopathological examinations revealed pseudohyphae/hyphae and yeast forms in the $C$ albicans infected rabbit lung tissues similar to findings in humans $[24,25]$. Blood samples on day 3 post-inoculation, when lung CT scans revealed evidence of pulmonary infection, revealed higher serum levels of PCT, sCD163, sTREM-1 and TNF- $\alpha$ in infected rabbits compared to the controls $(P<0.01)$, but no significant differences were found in CRP, IL-6, IL-8 and IL-10 levels or $\mathrm{PaO}_{2}$ between the groups.

To determine the specificity and sensitivity of markers, we performed ROC curve analyses and assessed the diagnostic values. Among the markers examined, sTREM-1 showed the highest sensitivity and specificity of 85 and $80 \%$ (cut-off value $45.88 \mathrm{pg} / \mathrm{mL}$ ), respectively, and the $\mathrm{AUC}_{\mathrm{ROC}}$ value was 0.882 (95\% CI: 0.922-0.976) indicating diagnostic accuracy. Also sCD163 showed a relatively high sensitivity and specificity of 80 and $75 \%$ (cut-off value $16.44 \mathrm{U} / \mathrm{mL}$ ), respectively, and the $\mathrm{AUC}_{\mathrm{ROC}}$ value was 0.814 (95\% CI: 0.678 0.950). Other markers did not demonstrate promising values in the ROC curve analyses.

The diagnostic values of sTREM- 1 and sCD163 have previously been extensively studied, and despite some contradictory reports sTREM-1 was successfully used as a marker for fungal or bacterial infection of the lungs $[7,19,20]$. Several studies have reported that the level of sCD163 in plasma was positively correlated with the severity of sepsis and proposed that sCDl63 could serve as a sepsis biomarker $[9,10]$.

However, in the present study disturbances in the levels of the sepsis markers by bacterial co-infections cannot be excluded, since no bacterial infection control was included and most studies on the biomarkers were carried out in the presence of bacterial infections. Nevertheless, the preliminary results of the present study may justify prospective monitoring of a human patient population. Another limitation of the present study was the small sample size. Further large scale studies will be required to confirm our findings and conclusions.

Taken together with other studies that STREM-1 and SCD163 were elevated in patients with bilateral lung infiltrates caused by bacterial or fungal pneumonia [19] and more generally in sepsis [10] and the elevation is consistent with an invasive disease either from pneumonia or other causes of sepsis which can be due to fungal pneumonia as demonstrated in this 
study or bacterial pneumonia as demonstrated in humans $[7,20]$, both factors might serve as marker for $\mathrm{CP}$ diagnosis.

\section{Conclusion}

We anticipate that measurement of both sTREM-1 and sCD163 levels in serum might, beside their function as indicators for bacterial infections, serve for the improvement of early $\mathrm{CP}$ diagnosis.

\begin{abstract}
Abbreviations
CP: Candida caused pneumonia; CRP: C-reactive protein; ICUs: intensive care unit; IFDs: invasive fungal diseases; IL-10: interleukin-10; IL-6: interleukin-6; IL8: interleukin-8; TNF-a: tumor necrosis factor-a; $\mathrm{PaO}_{2}$ : blood gas parameter; PCT: procalcitonin; SCD163: soluble hemoglobin-haptoglobin scavenger receptor; SIRS: Systemic inflammatory response syndrome; sTREM-1: soluble triggering receptor expressed on myeloid cells-1 ; WBC: white blood cell
\end{abstract}

\section{Acknowledgements}

Not applicable.

\section{Authors' contributions}

$\mathrm{GL}$ was responsible for the conception and design of the study. GL, CWang, CWu and LY were responsible for the acquisition and analysis of data; furthermore, GL, CWang and LY were in charge of statistical analysis. GL and CWu drafted the manuscript and CWang and JT revised it. All of the authors listed on the by-line have read and approved the manuscript.

\section{Funding}

This work was supported by the Project of Science and Technology Committee of Minhang District of Shanghai [grant number: 2014MHZ049]. This body played no part in in the design of the study or collection, analysis, interpretation of data or writing of this manuscript.

\section{Availability of data and materials}

The datasets used and/or analyzed during the current study are available from the corresponding author on reasonable request.

\section{Ethics approval and consent to participate}

All procedures involving animals were performed in accordance with the ethical standards of the participating institution and the Guidelines for the Humane Treatment of Laboratory Animals (Ministry of Science and Technology of the People's Republic of China, Policy No. 2006 398), and were approved by the Institutional Animal Care and Use Committee of Shanghai Fifth People's Hospital.

\section{Consent for publication}

Not applicable.

\section{Competing interests}

The authors declare that they have no competing interests.

\section{Author details}

'Department of Trauma-Emergency \& Critical Care Medicine, Shanghai Fifth People's Hospital, Fudan University, No. 128 Ruili Road, Shanghai 200240, China. ${ }^{2}$ Department of Respiratory, Taizhou Municipal Hospital, No. 381 East Zhongshan Road, Taizhou 318000, Zhejiang Province, China.

Received: 11 May 2018 Accepted: 25 July 2019

Published online: 06 August 2019

\section{References}

1. Vincent JL, Rello J, Marshall J, Silva E, Anzueto A, Martin CD, Moreno R, Lipman J, Gomersall C, Sakr Y, et al. International study of the prevalence and outcomes of infection in intensive care units. JAMA. 2009:302(21):2323-9.

2. De Pauw B, Walsh TJ, Donnelly JP, Stevens DA, Edwards JE, Calandra T, Pappas PG, Maertens J, Lortholary O, Kauffman CA, et al. Revised definitions of invasive fungal disease from the European Organization for Research and Treatment of cancer/invasive fungal infections cooperative group and the National Institute of Allergy and Infectious Diseases mycoses study group (EORTC/MSG) consensus group. Clin Infect Dis. 2008;46(12):1813-21.

3. Bouchon A, Dietrich J, Colonna M. Cutting edge: inflammatory responses can be triggered by TREM-1, a novel receptor expressed on neutrophils and monocytes. J Immunol. 2000;164(10):4991-5.

4. Gingras MC, Lapillonne H, Margolin JF. TREM-1, MDL-1, and DAP12 expression is associated with a mature stage of myeloid development. Mol Immunol. 2002;38(11):817-24.

5. Gibot S, Kolopp-Sarda MN, Bene MC, Bollaert PE, Lozniewski A, Mory F, Levy $B$, Faure GC. A soluble form of the triggering receptor expressed on myeloid cells-1 modulates the inflammatory response in murine sepsis. J Exp Med. 2004;200(11):1419-26.

6. Mahdy AM, Lowes DA, Galley HF, Bruce JE, Webster NR. Production of soluble triggering receptor expressed on myeloid cells by lipopolysaccharide-stimulated human neutrophils involves de novo protein synthesis. Clin Vaccine Immunol. 2006:13(4):492-5.

7. Porfyridis I, Plachouras D, Karagianni V, Kotanidou A, Papiris SA, Giamarellou H, Giamarellos-Bourboulis EJ. Diagnostic value of triggering receptor expressed on myeloid cells-1 and C-reactive protein for patients with lung infiltrates: an observational study. BMC Infect Dis. 2010;10:286.

8. Van Gorp H, Delputte PL, Nauwynck HJ. Scavenger receptor CD163, a Jackof-all-trades and potential target for cell-directed therapy. Mol Immunol. 2010;47(7-8):1650-60.

9. Madsen M, Moller HJ, Nielsen MJ, Jacobsen C, Graversen JH, van den Berg T, Moestrup SK. Molecular characterization of the haptoglobin.Hemoglobin receptor CD163. Ligand binding properties of the scavenger receptor cysteine-rich domain region. J Biol Chem. 2004;279(49):51561-7.

10. Feng L, Zhou X, Su LX, Feng D, Jia YH, Xie LX. Clinical significance of soluble hemoglobin scavenger receptor CD163 (sCD163) in sepsis, a prospective study. PLoS One. 2012;7(7):e38400.

11. Balc IC, Sungurtekin H, Gurses E, Sungurtekin U, Kaptanoglu B. Usefulness of procalcitonin for diagnosis of sepsis in the intensive care unit. Crit Care. 2003; $7(1): 85-90$

12. Brunkhorst FM, Al-Nawas B, Krummenauer F, Forycki ZF, Shah PM. Procalcitonin, C-reactive protein and APACHE II score for risk evaluation in patients with severe pneumonia. Clin Microbiol Infect. 2002:8(2):93-100.

13. Bozza FA, Salluh Jl, Japiassu AM, Soares M, Assis EF, Gomes RN, Bozza MT, Castro-Faria-Neto HC, Bozza PT. Cytokine profiles as markers of disease severity in sepsis: a multiplex analysis. Crit Care. 2007;11(2):R49.

14. Faix JD. Biomarkers of sepsis. Crit Rev Clin Lab Sci. 2013;50(1):23-36.

15. Lichtenstern C, Brenner T, Bardenheuer HJ, Weigand MA. Predictors of survival in sepsis: what is the best inflammatory marker to measure? Curr Opin Infect Dis. 2012;25(3):328-36.

16. Chuang TY, Chang HT, Chung KP, Cheng HS, Liu CY, Liu YC, Huang HH, Chou TC, Chang BL, Lee MR, et al. High levels of serum macrophage migration inhibitory factor and interleukin 10 are associated with a rapidly fatal outcome in patients with severe sepsis. Int J Infect Dis. 2014:20:13-7.

17. Clancy CJ, Nguyen ML, Cheng S, Huang H, Fan G, Jaber RA, Wingard JR, Cline C, Nguyen MH. Immunoglobulin G responses to a panel of Candida albicans antigens as accurate and early markers for the presence of systemic candidiasis. J Clin Microbiol. 2008;46(5):1647-54.

18. Naher $\mathrm{H}$, Al-dabagh N. Study the pathogenesis of Candida albicans in animal model. IOSR J Pharm Biol Sci. 2015:10:34-9.

19. Huh JW, Lim CM, Koh Y, Oh YM, Shim TS, Lee SD, Kim WS, Kim DS, Kim WD, Hong SB. Diagnostic utility of the soluble triggering receptor expressed on myeloid cells-1 in bronchoalveolar lavage fluid from patients with bilateral lung infiltrates. Crit Care. 2008;12(1):R6.

20. Shi JX, Li JS, Hu R, Li CH, Wen Y, Zheng H, Zhang F, Li Q. Diagnostic value of STREM-1 in bronchoalveolar lavage fluid in ICU patients with bacterial lung infections: a bivariate meta-analysis. PLoS One. 2013;8(5): e65436.

21. Naglik JR, Fidel PL Jr, Odds FC. Animal models of mucosal Candida infection. FEMS Microbiol Lett. 2008;283(2):129-39.

22. Hohl TM. Overview of vertebrate animal models of fungal infection. J Immunol Methods. 2014;410:100-12. 
23. Capilla J, Clemons KV, Stevens DA. Animal models: an important tool in mycology. Med Mycol. 2007;45(8):657-84.

24. Guarner J, Brandt ME. Histopathologic diagnosis of fungal infections in the 21st century. Clin Microbiol Rev. 2011;24(2):247-80.

25. Roden AC, Schuetz AN. Histopathology of fungal diseases of the lung. Semin Diagn Pathol. 2017;34(6):530-49.

\section{Publisher's Note}

Springer Nature remains neutral with regard to jurisdictional claims in published maps and institutional affiliations.

Ready to submit your research? Choose BMC and benefit from:

- fast, convenient online submission

- thorough peer review by experienced researchers in your field

- rapid publication on acceptance

- support for research data, including large and complex data types

- gold Open Access which fosters wider collaboration and increased citations

- maximum visibility for your research: over $100 \mathrm{M}$ website views per year

At $\mathrm{BMC}$, research is always in progress. 